H. Fujimoto

Nagoya Math. J.

Vol. 83 (1981), 153-181

\title{
REMARKS TO THE UNIQUENESS PROBLEM OF MEROMORPHIC MAPS INTO $P^{N}(C)$, IV
}

\author{
HIROTAKA FUJIMOTO
}

\section{§1. Introduction}

Let $H_{1}, H_{2}, \cdots, H_{N+2}$ be hyperplanes in $P^{N}(C)$ located in general position and $\nu_{1}, \nu_{2}, \cdots, \nu_{N+2}$ divisors on $C^{n}$. We consider the set $\mathscr{F}\left(H_{i}, \nu_{i}\right)$ of all non-degenerate meromorphic maps of $C^{n}$ into $P^{N}(C)$ such that the pullbacks $\nu\left(f, H_{i}\right)$ of the divisors $\left(H_{i}\right)$ on $P^{N}(C)$ by $f$ are equal to $\nu_{i}$ for any $i=1,2, \cdots, N+2$. In the previous paper [6], the author showed that $\mathscr{F}$ $:=\mathscr{F}\left(H_{i}, \nu_{i}\right)$ cannot contain more than $N+1$ algebraically independent maps. Relating to this, the following theorem will be proved.

THEOREM. The set $\mathscr{F}$ is finite.

We give here an example which shows that the number $\# \mathscr{F}$ of elements in $\mathscr{F}$ is not less than $(N+1)$ !. Take $N+1$ nowhere zero entire functions $h_{1}, \cdots, h_{N+1}$ such that $h_{i} / h_{j} \neq$ const if $i \neq j$, and define

$$
F:=h_{1}+h_{2}+\cdots+h_{N+1} \text {. }
$$

We consider hyperplanes

$$
\begin{aligned}
& H_{i}: w_{i}=0 \quad(1 \leqq i \leqq N+1) \\
& H_{N+2}: w_{1}+w_{2}+\cdots+w_{N+1}=0
\end{aligned}
$$

in $P^{N}(C)$ and divisors

$$
\begin{aligned}
& \nu_{i}=0 \quad(1 \leqq i \leqq N+1) \\
& \nu_{N+2}=\nu_{F}
\end{aligned}
$$

on $C^{n}$, where $w_{1}: w_{2}: \cdots: w_{N+1}$ are homogeneous coordinates on $P^{N}(C)$ and $\nu_{F}$ denotes the divisor defined by the zero-multiplicity of $F$. Then, $\mathscr{F}:=$ $\mathscr{F}\left(H_{i}, \nu_{i}\right)$ contains

$$
f^{\sigma}=h_{\sigma(1)}: h_{\sigma(2)}: \cdots: h_{\sigma(N+1)}
$$

Received September 14, 1979. 
for any permutation $\sigma=\left(\begin{array}{cccc}1 & 2 & \cdots & N+1 \\ \sigma(1) \sigma(2) & \cdots & \sigma(N+1)\end{array}\right)$. Therefore, $\sharp \mathscr{F} \geqq(N+1)$ !.

It is an interesting problem to ask if $\# \mathscr{F}$ is bounded from above by a constant depending only on $N$. But, the author cannot yet reply to it.

As an application of the above theorem, we shall show the following:

Let $f: C^{n} \rightarrow P^{N}(C)$ be a non-degenerate meromorphic map and $\gamma: C^{n}$ $\rightarrow C^{n}$ a biholomorphic map. If $\nu\left(f, H_{i}\right)(\gamma(z))=\nu\left(f, H_{i}\right)(z)$ for $N+2$ hyperplanes $H_{i}(1 \leqq i \leqq N+2)$ in general position, then there exists some positive integer $j_{0}$ such that $f \circ \gamma^{j_{0}}=f$, where $\gamma^{j_{0}}=\gamma \circ \gamma \circ \cdots \circ \gamma$ ( $j_{0}$-times).

Here, we cannot always take $j_{0}=1$. Consider a holomorphic map

$$
f(z):=e^{\sin (z /(N+1))}: e^{\sin ((z+2 \pi) /(N+1))}: \cdots: e^{\sin ((z+2 N \pi) /(N+1))}
$$

of $C$ into $P^{N}(C)$ and a biholomorphic map $\gamma: C \rightarrow C$ defined by $\gamma(z)=z+$ $2 \pi(z \in C)$. For hyperplanes $H_{i}(1 \leqq i \leqq N+2)$ defined by (1), we see

$$
\nu\left(f, H_{i}\right)(\gamma(z))=\nu\left(f, H_{i}\right)(z) \quad(1 \leqq i \leqq N+2),
$$

but $f(z+2 \pi) \neq f(z)$. In this case, we have to take $j_{0}=N+1$.

In the proof of the above theorem, the classical theorem of E. Borel ([1]) plays an essential role. We can generalize it to the case that meromorphic functions of order less than one are taken as coefficients. By the similar arguments as in the proof of the above theorem, we shall give some results on relations between meromorphic functions of order less than one and meromorphic functions with $\gamma$-invariant zeros and poles for a biholomorphic map $\gamma: C^{n} \rightarrow C^{n}$. One of them includes the following result as a special case.

TheOREM. Let $\varphi_{1}, \cdots, \varphi_{p}$ be meromorphic functions on $C$ of order less than one and $g_{1}, \cdots, g_{p}$ meromorphic functions on $C$ with $\nu_{g_{i}}(z+\omega)=\nu_{g_{i}}(z)$ for a non-zero constant $\omega$. If $\sum_{i=1}^{p} \varphi_{i} g_{i} \equiv 0$ and $\sum_{i \in I} \varphi_{i} g_{i} \neq \equiv 0$ for any proper subset $I$ of $\{1,2, \cdots, p\}$, then there exists some positive integer $j_{0}$ such that $h_{i_{1}} / h_{i_{2}}$ is a periodic function with period $j_{0} \omega$ for any $i_{1}$ and $i_{2}$.

By applying this, we shall generalize a recent result by Urabe-Yang in [11] and [12] which motivated the studies in this paper.

\section{§2. Preliminaries}

Let $\varphi(z)$ be a non-zero holomorphic function on a domain $D$ in $C^{n}$. For each point $a=\left(a_{1}, \cdots, a_{n}\right) \in D$, we expand $\varphi$ as a convergent series 


$$
\varphi\left(a_{1}+u_{1}, \cdots, a_{n}+u_{n}\right)=\sum_{m=0}^{\infty} P_{m}\left(u_{1}, \cdots, u_{n}\right)
$$

on a neighborhood of $a$, where $P_{m}$ is a homogeneous polynomial of degree $m$ or $P_{m} \equiv 0$. We define

$$
\nu_{\varphi}(a):=\min \left\{m ; P_{m}\left(u_{1}, \cdots, u_{n}\right) \neq 0\right\} .
$$

In case that $\varphi$ is meromorphic, taking non-zero holomorphic functions $\varphi_{1}$ and $\varphi_{2}$ in a neighborhood of $a$ such that $\varphi=\varphi_{1} / \varphi_{2}$ and

$$
\operatorname{codim}\left\{\varphi_{1}=\varphi_{2}=0\right\} \geqq 2,
$$

we define $\nu_{\varphi}^{0}=\nu_{\varphi_{1}}, \nu_{\varphi}^{\infty}=\nu_{\varphi_{2}}$ and $\nu_{\varphi}=\nu_{\varphi_{1}}-\nu_{\varphi_{2}}$, which are determined independently of the choices of $\varphi_{1}$ and $\varphi_{2}$. By definition, a divisor on $D$ is an integer-valued function on $D$ such that for any point $a \in D$ there is a nonzero meromorphic function $\varphi$ with $\nu=\nu_{\varphi}$ on a neighborhood of $a$ and the carrier of $\nu$ is an analytic set

$$
|\nu|:=\overline{\{z \in D ; \nu(z) \neq 0\}} \cap D .
$$

Definition 2.1. Let $\nu$ be a divisor on $C^{n}$. Take a positive constant $s$ arbitrarily. We define the counting function of $\nu$ by

$$
N(r, \nu):= \begin{cases}\frac{1}{W} \int_{s}^{r} \frac{d t}{t^{2 n-1}} \int_{|\nu| \cap \overline{B(t)}} \nu(z) v_{n-1}(z) & (r>s) \text { if } n>1 \\ \int_{s}^{r} \frac{1}{t}\left(\sum_{|z| \leqq t} \nu(z)\right) d t & (r>s) \text { if } n=1,\end{cases}
$$

where

$$
\begin{aligned}
v_{1}: & =\frac{\sqrt{-1}}{2}\left(d z_{1} \wedge d \bar{z}_{1}+\cdots+d z_{n} \wedge d \bar{z}_{n}\right) \\
v_{n-1}: & =\frac{1}{(n-1) !} v_{1} \wedge v_{1} \wedge \cdots \wedge v_{1} \quad((n-1) \text {-times }) \\
W: & =\frac{\pi^{n-1}}{(n-1) !} \\
B(t): & =\left\{z=\left(z_{1}, \cdots, z_{n}\right) ;\|z\|^{2}=\left|z_{1}\right|^{2}+\cdots+\left|z_{n}\right|^{2}<t^{2}\right\}
\end{aligned}
$$

and the integral over $|\nu| \cap \overline{B(t)}$ means that the integral over the manifold consisting of all regular points of $|\nu| \cap \overline{B(t)}$.

DeFinition 2.2. Let $\varphi$ be a non-zero meromorphic function on $C^{n}$. The order function of $\varphi$ is defined by 


$$
T(r, \varphi):=N\left(r, \nu_{\varphi}^{\infty}\right)+\frac{1}{\Phi(r)} \int_{S(r)} \log ^{+}|\varphi(z)| \sigma_{r}(z) \quad(r>s),
$$

where $\log ^{+} x=\max (\log x, 0), \Phi(r)=\left(2 \pi^{n} /(n-1) !\right) r^{2 n-1}, S(r):=\{z ;\|z\|=r\}$ and $\sigma_{r}$ denotes the area element of $S(r)$. In case $\varphi \equiv 0$, we define $T(r, \varphi)$ $\equiv 0$. The order of $\varphi$ is defined by

$$
\rho(\varphi):=\limsup _{r \rightarrow \infty} \frac{\log ^{+} T(r, \varphi)}{\log r}(\leqq+\infty) .
$$

As in the case of meromorphic functions on $C$, we can prove (2.3) If $\varphi$ is holomorphic, then

$$
\rho(\varphi)=\limsup _{r \rightarrow \infty} \frac{\log ^{+} \log ^{+} M(r, \varphi)}{\log r},
$$

where

$$
M(r, \varphi):=\max \{|\varphi(z)| ;\|z\|=r\} .
$$

For the proof, see W. Stoll [9].

(2.4) Let $\varphi_{1}$ and $\varphi_{2}$ be non-zero meromorphic functions on $C^{n}$ of order less than a positive number $\rho$. Then, $\varphi_{1}+\varphi_{2}, \varphi_{1}-\varphi_{2}, \varphi_{1} \varphi_{2}$ and $\varphi_{1} / \varphi_{2}$ are also of order less than $\rho$.

In fact, we can find some positive constants $M$ and $\rho_{0}$ with $0<\rho_{0}<\rho$ such that $T\left(r, \varphi_{i}\right) \leqq M r^{\rho_{0}}(i=1,2)$ for sufficiently large $r$. Putting $\psi:=$ $\varphi_{1} \pm \varphi_{2}$, or $\varphi_{1} \varphi_{2}^{ \pm 1}$, we have easily

$$
T(r, \psi) \leqq T\left(r, \varphi_{1}\right)+T\left(r, \varphi_{2}\right)+O(1) \leqq 2 M r^{\rho_{0}}+O(1)
$$
and so $\rho(\psi) \leqq \rho_{0}<\rho$.

Definition 2.5. Let $\nu$ be a divisor on $C^{n}$. We define the order of $\nu$ by

$$
\rho(\nu):=\limsup _{r \rightarrow \infty} \frac{\log ^{+} N(r, \nu)}{\log r} .
$$

Take a pure $(n-1)$-dimensional analytic set in $C^{n}$. We can define a divisor $\nu_{V}$ on $C^{n}$ such that $\left|\nu_{V}\right|=V$ and $\nu_{V}(z)=1$ for any regular point $z$ of $V$. We call the order of $\nu_{V}$ the order of $V$.

(2.6) Let $\varphi$ be a non-constant meromorphic function on $C^{n}$. Then, for any $a \in C$

$$
N\left(r, \nu_{\varphi-a}^{0}\right) \leqq T(r, \varphi)+O(1)
$$


For the proof, see H. Fujimoto [2], pp. 34-35.

(2.7) For a divisor $\nu$ on $C^{n}$ there exists a meromorphic function $\varphi$ on $C^{n}$ such that $\nu_{\varphi}=\nu$ and $\rho(\varphi) \leqq \rho(\nu)$.

For the proof, see W. Stoll [9].

(2.8) Let $\varphi$ be a holomorphic function on $C^{n}$ and $\omega=\left(\omega_{1}, \cdots, \omega_{n}\right) \in C^{n}$ - \{0\}. We define a holomorphic function $\varphi_{\omega}$ on $C$ by $\varphi_{\omega}(z):=\varphi(z \omega)$, where $z \omega=\left(z \omega_{1}, \cdots, z \omega_{n}\right)$. Then, $\rho\left(\varphi_{\omega}\right) \leqq \rho(\varphi)$.

This is an immediate consequence of (2.3), because

$$
M\left(r, \varphi_{\omega}\right)=\max _{\|z\|=r}|\varphi(z \omega)| \leqq M(\|\omega\| r, \varphi) .
$$

(2.9) If $h(z)$ is a nowhere zero non-constant holomorphic function on $C^{n}$, then $\rho(h) \geqq 1$.

This is well-known for the case $n=1$ (c.f., [8]). Let $n \geqq 2$. We can take a point $\omega \in C^{n}-\{0\}$ such that $h_{\omega}(z):=h(z \omega) \not \equiv$ const. By (2.8), we see

$$
\rho(h) \geqq \rho\left(h_{\omega}\right) \geqq 1 \text {. }
$$

We denote the set of all nowhere zero holomorphic functions on $C^{n}$ by $\boldsymbol{H}^{*}$ and the set of all meromorphic functions of order less than one by $\Phi_{0}$. And, for $h, h^{\prime} \in H^{*}$, we mean by $h \sim h^{\prime}$ and $h \not h^{\prime}$ that $h / h^{\prime} \equiv$ const and $h / h^{\prime} \not \equiv$ const respectively.

Now, we give a generalization of the classical theorem of E. Borel.

TheOREM 2.10. Let $h_{1}, \cdots, h_{p} \in \boldsymbol{H}^{*}$ and $\varphi_{1}, \cdots, \varphi_{p} \in \Phi_{0}$. If $h_{i} \not h_{\text {j }}$ for any $i, j$ with $i \neq j$ and

$$
\varphi_{1} h_{1}+\varphi_{2} h_{2}+\cdots+\varphi_{p} h_{p} \equiv 0,
$$

then

$$
\varphi_{1} \equiv \varphi_{2} \equiv \cdots \equiv \varphi_{p} \equiv 0 \text {. }
$$

Proof. This is a well-known fact if $n=1$ (c.f., for example, [7], p. 100). Let us consider the case $n \geqq 2$. To prove Theorem 2.10 by induction on $p$, it suffices to show that at least one $\varphi_{i}$ vanishes. Assume that $\varphi_{i} \not \equiv 0$ for any $i$. For a point $\omega \in C^{n}-\{0\}$, we define $\left(\varphi_{i}\right)_{\omega}(z):=\varphi_{i}(z \omega)$ and $\left(h_{i}\right)_{\omega}(z)$ $:=h_{i}(z \omega)$. We see easily

$$
\bigcup_{i}\left\{\omega ;\left(\varphi_{i}\right)_{\omega} \equiv 0\right\} \cup \bigcup_{i<j}\left\{\omega ; \frac{\left(h_{i}\right)_{\omega}}{\left(h_{j}\right)_{\omega}} \equiv \frac{h_{i}(0)}{h_{j}(0)}\right\} \cong C^{n}-\{0\} .
$$


Therefore, we can find some $\omega \in C^{n}-\{0\}$ such that $\left(\varphi_{i}\right)_{\omega} \neq \equiv 0(1 \leqq i \leqq p)$ and $\left(h_{i}\right)_{\omega} /\left(h_{j}\right)_{\omega} \neq \equiv$ const $(1 \leqq i<j \leqq p)$. The assumption (2) gives the identity

$$
\left(\varphi_{1}\right)_{\omega}\left(h_{1}\right)_{\omega}+\cdots+\left(\varphi_{p}\right)_{\omega}\left(h_{p}\right)_{\omega} \equiv 0 .
$$

This contradicts Theorem 2.10 for the case $n=1$. We have thus the desired result.

Corollary 2.11. Let $h_{1}, \cdots, h_{p} \in H^{*}$ and assume that

$$
h_{1}^{\ell_{1}} h_{2}^{\ell_{2}} \cdots h_{p}^{\ell_{p}} \not \equiv \text { const }
$$

for any non-zero vector $\left(\ell_{1}, \ell_{2}, \cdots, \ell_{p}\right)$ of integers. If finitely many $\varphi_{\ell_{1} \ldots \ell_{p}} \in \Phi_{0}$ satisfy

$$
\sum_{\left(\ell_{1}, \ldots, \ell_{p}\right)} \varphi_{\ell_{1} \cdots \ell_{p}} h_{1}^{\ell_{1}} \cdots h_{p}^{\ell_{p}} \equiv 0,
$$

then $\varphi_{\ell_{1} \ldots \ell_{p}} \equiv 0$ for any $\left(\ell_{1}, \cdots, \ell_{p}\right)$.

Proof. Since $h_{1}^{\ell_{1}} \cdots h_{p}^{\ell_{p}} \in \boldsymbol{H}^{*}$ and

$$
h_{1}^{\ell_{1}} \cdots h_{p}^{\ell_{p}} \nsim h_{1}^{m_{1}} \cdots h_{p}^{m_{p}}
$$

whenever $\left(\ell_{1}, \cdots, \ell_{p}\right) \neq\left(m_{1}, \cdots, m_{p}\right)$, Corollary 2.11 is a direct result of Theorem 2.10.

Corollary 2.12. Let $h_{1}, \cdots, h_{p} \in \boldsymbol{H}^{*}$ and $\varphi_{1}, \cdots, \varphi_{p} \in \Phi_{0}$ satisfy the condition that $\varphi_{i} \not \equiv 0$ and

$$
\varphi_{1} h_{1}+\varphi_{2} h_{2}+\cdots+\varphi_{p} h_{p} \equiv 0 .
$$

Consider the partition of indices

$$
\{1,2, \cdots, p\}=I_{1} \cup I_{2} \cup \cdots \cup I_{a}
$$

such that, for any $i \in I_{\alpha}$ and $i^{\prime} \in I_{\alpha^{\prime}}, h_{i} \sim \cdot h_{i^{\prime}}$ if $\alpha=\alpha^{\prime}$, and $h_{i} \nsim h_{i^{\prime}}$ if $\alpha \neq \alpha^{\prime}$. Then, for any $\alpha$,

$$
\sum_{i \in I_{\alpha}} \varphi_{i} h_{i} \equiv 0 .
$$

Proof. Taking an index $i_{\alpha} \in I_{\alpha}$ for each $\alpha$, we define

$$
\psi_{\alpha}:=\sum_{i \in I_{\alpha}} \varphi_{i} h_{i} / h_{i_{\alpha}} .
$$

Then, $\psi_{\alpha} \in \Phi_{0}, h_{i \alpha} \not h_{i^{\prime}}$ if $\alpha \neq \alpha^{\prime}$, and $\sum_{\alpha} \psi_{\alpha} h_{i_{\alpha}} \equiv 0$. By Theorem 2.10, we have $\psi_{1} \equiv \cdots \equiv \psi_{a} \equiv 0$. This gives Corollary 2.12. 


\section{§ 3. Basic lemmas}

Take $h_{i j} \in \boldsymbol{H}^{*}$ and $\varphi_{i j} \in \Phi_{0}$ with $\varphi_{i j} \not \equiv 0$, where $i=1,2, \cdots, p$ and $j=$ $1,2, \cdots$. Defining $f_{i j}:=\varphi_{i j} h_{i j}$, we consider a matrix

$$
\mathscr{M}:=\left(f_{i j} ; i=1,2, \cdots, p, j=1,2, \cdots\right)
$$

with $p$ rows and countably many columns.

LEMMA 3.1. If we perform the operations (a) changing the order of the indices $i=1,2, \cdots, p$, (b) replacing a suitable subsequence of the indices $j$ 's by $j=1,2, \cdots$ and (c) multiplying each row and each column by a common element of $H^{*}$, then $\mathscr{M}=\left\{f_{i j}:=\varphi_{i j} h_{i j}\right\}$ may be assumed to satisfy the conditions;

(i) $h_{i j_{1}} \nsucc h_{i j_{2}}$ if $1 \leqq i \leqq r$ and $j_{1} \neq j_{2}$,

(ii) $h_{i j} \equiv$ const for any $j$ if $r+1 \leqq i \leqq p$,

where $0 \leqq r<p$ and $r=0$ means that $h_{i j} \equiv$ const for any $i, j$.

Proof. Dividing $h_{i j}(1 \leqq i \leqq p)$ by $h_{p j}$, we may assume $h_{p j} \equiv 1$ for each $j$. We consider the smallest integer $r$ such that, after performing the operations (a) $\sim$ (c), the condition (ii) is satisfied, where we may assume $0<r<p$. Then, for any $i=1,2, \cdots, r$ and $j=1,2, \cdots$, there are only finitely many $j^{\prime}$ such that $h_{i j} \sim h_{i j^{\prime}}$. Because, if not, we have some $i_{0}$ with $1 \leqq i_{0} \leqq r$ and $j_{0}$ such that $h_{i_{0} j_{0}} \sim h_{i_{0} j}$ for infinitely many $j$. After performing suitable operations $(\mathrm{a}) \sim(\mathrm{c})$, we may assume $h_{r j} \equiv$ const, which contradicts the property of $r$. We can choose indices $j_{1}, j_{2}, \cdots$ such that $j_{k-1}<j_{k}$ and, for any $i=1,2, \cdots, r$,

$$
h_{i j_{k}} \nsim h_{i j_{1}}, h_{i j_{k}} \nsim h_{i j_{2}}, \cdots, h_{i j_{k}} \nsim h_{i j_{k-1}} .
$$

If we replace the indices $j=j_{1}, j_{2}, \ldots$ by $j=1,2, \cdots$, we obtain the conclusion of Lemma 3.1 .

Lemma 3.2. Assume that $\mathscr{M}=\left\{f_{i j}:=\varphi_{i j} h_{i j}\right\}$ satisfies the conclusion of Lemma 3.1 and, furthermore, for any $j_{1}, \cdots, j_{p}$,

$$
\operatorname{det}\left(f_{i j} ; i=1,2, \cdots, p, j=j_{1}, j_{2}, \cdots, j_{p}\right) \equiv 0 .
$$

If for any $j$ there exist indices $j_{r+1}^{*}, \cdots, j_{p}^{*}$ such that $j<j_{r+1}^{*}<\cdots<j_{p}^{*}$ and

$$
\operatorname{det}\left(f_{i j} ; i=r+1, \cdots, p, j=j_{r+1}^{*}, \cdots, j_{p}^{*}\right) \not \equiv 0,
$$

then

$$
\operatorname{det}\left(f_{i j} ; i=1,2, \cdots, r, j=j_{1}, j_{2}, \cdots, j_{r}\right) \equiv 0
$$


for any $j_{1}, j_{2}, \cdots, j_{r}$.

Proof. If $r=0$, we have nothing to prove. Let $r>0$. The set $\boldsymbol{H}^{*}$ can be regarded as a multiplicative group and includes $C^{*}:=C-\{0\}$ as a subgroup. The factor group $G:=\boldsymbol{H}^{*} / C^{*}$ is a torsionfree abelian group. We denote the class in $G$ containing an element $h \in \boldsymbol{H}^{*}$ by $[h]$. We choose finitely many or countably many elements $\eta_{1}, \eta_{2}, \cdots, \eta_{\tau}, \cdots$ in $\boldsymbol{H}^{*}$ such that

(i) $\left[\eta_{1}\right],\left[\eta_{2}\right], \cdots,\left[\eta_{\tau}\right], \cdots$ are linearly independent over $\boldsymbol{Z}$ and

(ii) each $h_{i j}$ can be represented as

$$
h_{i j}=c_{i j} \eta_{1}^{\ell_{i j}^{1}} \eta_{2}^{\ell_{i j}^{2}} \cdots \eta_{\tau}^{\ell_{i j}^{\tau}} \ldots \text {, }
$$

where $c_{i j} \in C^{*}, \ell_{i j}^{\tau} \in Z$ and $\ell_{i j}^{\tau}=0$ except finitely many $\tau$ for each $(i, j)$. Define

$$
\ell_{i j}=\left(\ell_{i j}^{1}, \ell_{i j}^{2}, \cdots, \ell_{i j}^{\tau}, \cdots\right) .
$$

By the assumption, $\ell_{i j_{1}} \neq \ell_{i j_{2}}$ if $1 \leqq i \leqq r$ and $j_{1} \neq j_{2}$, and $\ell_{i j}=0$ for any $j$ if $r+1 \leqq i \leqq p$.

Now, assume

$$
\operatorname{det}\left(f_{i j} ; 1 \leqq i \leqq r, j=j_{1}, \cdots, j_{r}\right) \not \equiv 0
$$

for some $j_{1}, \cdots, j_{r}$ with $j_{1}<\cdots<j_{r}$. Then, we can prove

(3.3) There exist indices $j_{r+1}, \cdots, j_{p}$ with $j_{r+1}<\cdots<j_{p}$ such that, for any $s=r+1, \cdots, p$,

(A) $\operatorname{rank}\left(f_{i j} ; i=r+1, \cdots, p, j=j_{r+1}, \cdots, j_{s}\right)=s-r$,

(B) $\ell_{i j_{s}} \neq \ell_{\sigma_{1} j_{1}}+\cdots+\ell_{o_{s-1} j_{s-1}}-\left(\ell_{\tau_{1} j_{1}}+\cdots+\ell_{\tau_{s-1} j_{s-1}}\right)$

whenever $1 \leqq i \leqq r$ and $\sigma_{1}, \cdots, \sigma_{s-1}, \tau_{1}, \cdots, \tau_{s-1} \in\{1,2, \cdots, p\}$.

To see this, we first choose $j_{r+1}$ with $j_{r}<j_{r+1}$ such that

$$
\left(f_{r+1 j_{r+1}}, \cdots, f_{p_{r+1}}\right) \not \equiv(0, \cdots, 0) \text {. }
$$

Let $j_{r+1}, \cdots, j_{s-1}$ be chosen so that $j_{r+1}<\cdots<j_{s-1}$ and they satisfy the conditions (A) and (B). By $m$ we denote the field of all meromorphic functions on $\boldsymbol{C}^{n}$. If we set $\boldsymbol{f}_{j}={ }^{t}\left(f_{r+1 j}, \cdots, f_{p j}\right) \in \mathfrak{m}^{p-r}$, then $\boldsymbol{f}_{j_{r+1}}, \cdots, \boldsymbol{f}_{j_{s-1}}$ are linearly independent over $\mathfrak{m}$. Therefore, there are at most $s-r-1$ linearly independent elements $\boldsymbol{g}^{\prime} \mathbf{s}$ in $\mathfrak{m}^{p-r}$ such that

$$
\operatorname{rank}\left(f_{j_{r+1}}, \cdots, f_{j_{s-1}}, g\right) \leqq s-r-1 \text {. }
$$


On the other hand, for any $j$, there are indices $j_{r+1}^{*}, \cdots, j_{p}^{*}$ with $j<j_{r+1}^{*}$ $<\cdots<j_{p}^{*}$ satisfying the condition (4). We can choose an index $j_{s}$ among $j_{r+1}^{*}, \cdots, j_{p}^{*}$ such that

$$
\operatorname{rank}\left(f_{j_{r+1}}, \cdots, f_{j_{s-1}}, f_{j_{s}}\right)=s-r .
$$

Accordingly, there are infinitely many $j_{s}$, s satisfying the condition (A). Next, let us examine the condition (B). The set

$$
\begin{array}{r}
\left\{\ell_{\sigma_{1} j_{1}}+\cdots+\ell_{\sigma_{s-1} j_{s-1}}-\left(\ell_{\tau_{1} j_{1}}+\cdots+\ell_{\tau_{s-1} j_{s-1}}\right) ;\right. \\
\left.1 \leqq \sigma_{1}, \cdots, \sigma_{s-1}, \tau_{1}, \cdots, \tau_{s-1} \leqq p\right\}
\end{array}
$$

is finite. Since $\ell_{i j_{1}} \neq \ell_{\imath j_{2}}$ if $1 \leqq i \leqq r$ and $j_{1} \neq j_{2}$, there are only finitely many $j_{s}$ 's such that

$$
\ell_{i j_{s}}=\ell_{\sigma_{1} j_{1}}+\cdots+\ell_{\sigma_{s-1} j_{s-1}}-\left(\ell_{\tau_{1} j_{1}}+\cdots+\ell_{\tau_{s-1} j_{s-1}}\right)
$$

for some $i \in\{1,2, \cdots, r\}$ and $\sigma_{1}, \cdots, \sigma_{s-1}, \tau_{1}, \cdots, \tau_{s-1} \in\{1,2, \cdots, p\}$. Consequently, we can find infinitely many $j_{s}$ 's satisfying the conditions (A) and (B). And, we have the desired indices $j_{r+1}, \cdots, j_{p}$ inductively.

Now we go back to the proof of Lemma 3.2. Let $j_{1}, \ldots, j_{p}$ satisfy the conditions (6) and (A), (B) of (3.3). We denote by $S_{p}$ the symmetric group of all permutations of $p$ letters $1,2, \cdots, p$ and set

$$
\begin{aligned}
S_{p}^{(1)}: & =\left\{\sigma=\left(\begin{array}{ccc}
12 & \cdots & p \\
\sigma_{1} \sigma_{2} & \cdots & \sigma_{p}
\end{array}\right) ; 1 \leqq \sigma_{i} \leqq r \text { for } i=1,2, \cdots, r\right\} \\
S_{p}^{(2)}: & =S_{p}-S_{p}^{(1)} .
\end{aligned}
$$

The assumption (3) may be rewritten

$$
\sum_{\sigma \in S_{p}^{(1)}} \psi_{o} h_{\sigma}+\sum_{\sigma \in S_{p}^{(2)}} \psi_{\sigma} h_{\sigma} \equiv \sum_{\sigma \in S_{p}} \psi_{o} h_{\sigma} \equiv 0
$$

where, for $\sigma=\left(\begin{array}{cccc}1 & 2 & \cdots & p \\ \sigma_{1} \sigma_{2} & \cdots & \sigma_{p}\end{array}\right) \in S_{p}$

$$
\begin{aligned}
& \psi_{o}:=\operatorname{sgn}(\sigma) \varphi_{\sigma_{1} j_{1}} \varphi_{\sigma_{2} j_{2}} \cdots \varphi_{\sigma_{p j}} \in \Phi_{0} \\
& h_{\sigma}:=h_{\sigma_{1} j_{1}} h_{\sigma_{2} j_{2}} \cdots h_{\sigma_{p} j_{p}} \in \boldsymbol{H}^{*} .
\end{aligned}
$$

We shall show $h_{\sigma} \not h_{\tau}$ whenever $\sigma \in S_{p}^{(1)}$ and $\tau \in S_{p}^{(2)}$. On the contrary, suppose $h_{\sigma} \sim h_{\tau}$ for some $\sigma \in S_{p}^{(1)}$ and $\tau \in S_{p}^{(2)}$. By substituting (5) and observing the exponents, we get

$$
\ell_{\sigma_{1} j_{1}}+\cdots+\ell_{\sigma_{p} j_{p}}=\ell_{\tau_{1} j_{1}}+\cdots+\ell_{\tau_{p} j_{p}} .
$$


By definition, $\left\{\sigma_{r+1}, \cdots, \sigma_{p}\right\}=\{r+1, \cdots, p\}$, and $\left\{\tau_{r+1}, \cdots, \tau_{p}\right\} \neq\{r+1, \cdots, p\}$. Choose index $s$ with $r+1 \leqq s \leqq p$ such that $\tau_{s} \notin\{r+1, \cdots, p\}$ and $\tau_{s+1}, \cdots$, $\tau_{p} \in\{r+1, \cdots, p\}$. Since $\ell_{i j}=0$ for any $j$ and $i=r+1, \cdots, p$,

$$
\ell_{\tau_{s} j_{s}}=\ell_{\sigma_{1} j_{1}}+\cdots+\ell_{\sigma_{s-1} j_{s-1}}-\left(\ell_{\tau_{1} j_{1}}+\cdots+\ell_{\tau_{s-1} j_{s-1}}\right) \text {. }
$$

This contradicts the condition (B) of (3.3). We now apply Corollary (2.12) to the indentity (7). From the above shown fact, we can conclude

$$
\sum_{\sigma \in S_{p}^{(1)}} \psi_{\sigma} h_{\sigma}=0
$$

On the other hand,

$$
\begin{aligned}
& \sum_{\sigma \in S_{p}^{(1)}} \psi_{\sigma} h_{\sigma} \\
& =\left(\sum_{\sigma=\left(\begin{array}{c}
1 \\
\sigma_{102} \ldots r \\
\sigma_{1} \cdots \sigma_{r}
\end{array}\right)} \operatorname{sgn}(\sigma) f_{\sigma_{1} j_{1}} \cdots f_{\sigma_{r} j_{r}}\right) \times\left(\sum_{\tau=\left(\begin{array}{c}
r+1 \ldots p \\
\tau_{r+1} \cdots \tau_{p}
\end{array}\right)} \operatorname{sgn}(\tau) f_{\tau_{r+1} j_{r+1}} \cdots f_{\tau_{p} j_{p}}\right) \\
& =\operatorname{det}\left(f_{i j} ; i=1,2, \cdots, r, j=j_{1}, \cdots, j_{r}\right) \\
& \times \operatorname{det}\left(f_{i j} ; i=r+1, \cdots, p, j=j_{r+1}, \cdots, j_{p}\right) \text {. }
\end{aligned}
$$

This does not vanish because of (6) and the conclusion of (3.3). The proof of Lemma 3.2 is completed.

Lemma 3.4. As in Lemma 3.2, suppose that $\mathscr{M}=\left\{f_{i j}\right\}$ satisfies the condition (3). Then, after performing the operations (b) and (c) of Lemma 3.1, we can find indices $i_{1}, \cdots, i_{m}$ with $1 \leqq i_{1}<\cdots<i_{m} \leqq p$ such that

$$
h_{i j} \equiv \text { const }
$$

for $i=i_{1}, \cdots, i_{m}$ and $j=1,2, \cdots$, and

$$
\operatorname{det}\left(f_{i j} ; i=i_{1}, \cdots, i_{m}, j=j_{1}, \cdots, j_{m}\right) \equiv 0
$$

for any $j_{1}, j_{2}, \cdots, j_{m}$.

Proof. This is shown by induction on $p$. If $p=2$, the conclusion is trivial. Suppose that Lemma 3.4 is true for the case $\leqq p-1$. We may assume that $\mathscr{M}$ satisfies the conditions (i) and (ii) of Lemma 3.1. If the assumption of Lemma 3.2 is satisfied, then we can apply the induction hypothesis to functions $f_{i j}$ for $i=1,2, \cdots, r$ and $j=1,2, \cdots$ and so obtain the desired conclusion. Otherwise, there is some $j_{0}$ such that

$$
\operatorname{det}\left(f_{i j} ; i=r+1, \cdots, p, j=j_{r+1}, \cdots, j_{p}\right) \equiv 0
$$


for any $j_{r+1}, \cdots, j_{p}$ larger than $j_{0}$. If we replace $j_{0}+1, j_{0}+2, \cdots$ by $1,2, \cdots$ and set $i_{1}=r+1, \cdots, i_{m}=p$, we have also the desired conclusion.

\section{§4. The main theorem}

Firstly, we shall recall some notation and terminologies. Let $f$ be a meromorphic map of $C^{n}$ into $P^{N}(C)$ which is non-degenerate, that is, the image of $f$ is not included in any hyperplane in $P^{N}(C)$. For arbitrarily fixed homogeneous coordinates $w_{1}: w_{2}: \cdots: w_{N+1}, f$ has a reduced representation

$$
f=f_{1}: f_{2}: \cdots: f_{N+1},
$$

where $f_{1}, \cdots, f_{N+1}$ are holomorphic on $C^{n}$ and satisfy the condition

$$
\operatorname{codim}\left\{f_{1}=f_{2}=\cdots=f_{N+1}=0\right\} \geqq 2 .
$$

Take a hyperplane

$$
H: a^{1} w_{1}+a^{2} w_{2}+\cdots+a^{N+1} w_{N+1}=0
$$

in $P^{N}(C)$. Regarding it as a divisor on $P^{N}(C)$, we define its pull-back $\nu(f, H)$ by

$$
\nu(f, H)(z)=\nu_{F}(z) \quad\left(z \in C^{n}\right)
$$

with a holomorphic function

$$
F:=a^{1} f_{1}+a^{2} f_{2}+\cdots+a^{N+1} f_{N+1} .
$$

Now, we consider hyperplanes $H_{1}, H_{2}, \cdots, H_{N+2}$ in $P^{N}(C)$ located in general position and divisors $\nu_{1}, \nu_{2}, \cdots, \nu_{N+2}$ on $C^{n}$. As is stated in $\S 1$, we denote by $\mathscr{F}:=\mathscr{F}\left(H_{i}, \nu_{i}\right)$ the set of all non-degenerate meromorphic maps of $C^{n}$ into $P^{N}(C)$ such that $\nu\left(f, H_{i}\right)=\nu_{i}$ for $i=1,2, \cdots, N+2$. The main Theorem is the following.

TheOREM 4.1. The set $\mathscr{F}$ contains at most finitely many maps.

For the proof, we identify $P^{N}(C)$ with the subspace

$$
\left\{w_{1}+w_{2}+\cdots+w_{N+2}=0\right\}
$$

in $P^{N+1}(C)$, where $w_{1}: \cdots: w_{N+2}$ are homogeneous coordinates on $P^{N+1}(C)$. Moreover, by a suitable change of coordinates, we may assume

$$
H_{i}=\left\{w_{i}=0\right\} \cap P^{N}(C) \quad(1 \leqq i \leqq N+2) .
$$


Suppose that $\mathscr{F}$ contains infinitely many mutually distinct maps $f^{1}$, $f^{2}, \cdots, f^{\prime}, \cdots$. Using the above coordinates, we take a reduced representation

$$
f^{j}=f_{1}^{j}: f_{2}^{j}: \cdots: f_{N+2}^{j}
$$

of each $f^{j}$. By (2.7) there exist entire functions $k_{i}$ with $\nu_{k_{i}}=\nu_{i}$ for $i=$ $1,2, \cdots, N+2$. Since $\nu\left(f^{\jmath}, H_{i}\right)=\nu_{i}$,

$$
h_{i j}:=f_{i}^{j} / k_{i} \in H^{*} .
$$

They satisfy $\sum_{i=1}^{N+2} h_{i j} k_{i} \equiv 0$ for any $j=1,2, \cdots$.

Lemma 4.2. Let $h_{i j} \in H^{*}(1 \leqq i \leqq p, j=1,2, \cdots)$ and $k_{i}(1 \leqq i \leqq p)$ be non-zero entire functions satisfying

$$
\sum_{i=1}^{p} h_{i j} k_{i}=0
$$

for any $j$ and, furthermore,

$$
\sum_{i \in I} h_{i j} k_{i} \not \equiv 0
$$

for any $j$ and any proper subset $I$ of $\{1,2, \cdots, p\}$. Then, there exists a subsequence $\left\{j_{1}, j_{2}, \cdots\right\}$ of $\{1,2, \cdots\}$ such that $h_{i j} \equiv$ const for any $i=1,2, \cdots, p$ and $j=j_{1}, j_{2}, \ldots$ after dividing each row and each column of $\left(h_{i j}\right)$ by a common element of $\boldsymbol{H}^{*}$.

Proof. The proof is given by induction on $p$. If $p=2$, we have easily Lemma 4.2 because $h_{1 j_{1}} / h_{2 j_{1}}=h_{1 j_{2}} / h_{2 j_{2}}$ for any $j_{1}$ and $j_{2}$. Suppose that Lemma 4.2 is true in the case $\leqq p-1$. Eliminating $k_{\imath}$ from the identities (8), we get

$$
\operatorname{det}\left(h_{i j} ; i=1,2, \cdots, p, j=j_{1}, j_{2}, \cdots, j_{p}\right) \equiv 0
$$

for any $j_{1}, j_{2}, \cdots, j_{p}$. We now apply Lemma 3.4. After performing the operations (a) $\sim$ (c) of Lemma 3.1, it may be assumed that $h_{i j} \equiv$ const for any $j$ and $i$ with $r+1 \leqq i \leqq p$, and

$$
\operatorname{det}\left(h_{i j} ; i=r+1, \cdots, p, j=j_{r+1}, \cdots, j_{p}\right) \equiv 0
$$

for any $j_{r+1}, \cdots, j_{p}$, where $0 \leqq r \leqq p-1$. Suppose that $r>0$. We may assume that $h_{1 j_{1}} \neq h_{1 j_{2}}$ for any $j_{1}, j_{2}$ with $j_{1} \neq j_{2}$ by the same argument as in the proof of Lemma 3.1. When we multiply the $i$-th row of $\left(h_{i j}\right)$ by a function in $H^{*}$, (8) does not alter if we replace $k_{i}$ by one divided by the same function. When we multiply the $j$-th column of $\left(h_{i j}\right)$ by a function 
in $H^{*}$, (8) remains valid if (8) is replaced by one divided by the same function. Therefore, we may assume in the original identities (8) that $h_{1 j_{1}} \nsim$ $h_{1 j_{2}}$ if $j_{1} \neq j_{2}$ and $h_{i j} \equiv$ const if $r+1 \leqq i \leqq p$.

Since

$$
\operatorname{rank}\left(h_{i j} ; r+1 \leqq i \leqq p, j=1,2, \cdots\right)<p-r,
$$

we can find a non-zero vector $\left(\lambda_{r+1}, \cdots, \lambda_{p}\right) \in C^{p-r}$ such that

$$
\sum_{i=r+1}^{p} \lambda_{i} h_{i j}=0 \quad(j=1,2, \cdots) .
$$

Take a regular matrix $A=\left(a_{i j} ; r+1 \leqq i, j \leqq p\right)$ of order $p-r$ such that $a_{i p}=\lambda_{i}(r+1 \leqq i \leqq p)$. Define functions $k_{r+1}^{*}, \cdots, k_{p}^{*}$ by the relations

$$
k_{i}=\sum_{\ell=r+1}^{p} a_{i \ell} k_{\ell}^{*} \quad(r+1 \leqq i \leqq p) .
$$

Then, (8) becomes

$$
\sum_{i=1}^{r} h_{i j} k_{i}+\sum_{\ell=r+1}^{p-1} h_{\ell j}^{*} k_{\ell}^{*}=0
$$

where

$$
h_{\ell j}^{*}:=\sum_{i=r+1}^{p} a_{i \ell} h_{i j} \in \boldsymbol{C} .
$$

For convenience' sake, we set $k_{i}^{*}:=k_{i}$ and $h_{i j}^{*}:=h_{i j}$ for $i=1,2, \cdots, r$. After changing the indices $j$ 's suitably, we can take a subset $I$ of $\{1,2, \cdots$, $p-1\}$ such that $1 \in I$,

$$
\sum_{i \in I} h_{i j}^{*} k_{i}^{*} \equiv 0
$$

and for any proper subset $I^{\prime}$ of $I$ and any $j=1,2, \cdots$,

$$
\sum_{i \in I^{\prime}} h_{i j}^{*} k_{i}^{*} \not \equiv 0 \text {. }
$$

By the assumption (9), there is some $i_{0} \in I \cap\{r+1, \cdots, p\}$. Since $\sharp I \leqq p$ -1 , by the induction hypothesis we see $h_{i j}^{*} \equiv$ const for $i \in I$ and $j=$ $1,2, \cdots$ after suitable changes of indices and $h_{i j}^{*}$. This is a contradiction. Because, $h_{i_{0} j}^{*} \equiv$ const for any $j$ and $h_{1 j_{1}}^{*} \not h_{1 j_{2}}^{*}$ for any $j_{1}, j_{2}$ with $j_{1} \neq j_{2}$. Consequently, $r=0$ and we have Lemma 4.2 .

Proof of Theorem 4.1. As a consequence of Lemma 4.2, changing $k_{i}$ suitably, taking a suitable subsequence of the indices $j$ 's and choosing a 
suitable reduced representation of each $f^{j}$, we may assume $h_{i j} \equiv$ const for any $i, j$, and particularly

$$
h_{11} \equiv h_{21} \equiv \cdots \equiv h_{p 1} \equiv 1,
$$

where $p=N+2$. Then

$$
f^{j}=h_{1 j} f_{1}^{1}: h_{2 j} f_{2}^{1}: \cdots: h_{N+2 j} f_{N+2}^{1}
$$

for $j=2,3, \cdots$, which satisfy

$$
h_{1 j} f_{1}^{1}+h_{2 j} f_{2}^{1}+\cdots+h_{N+2 j} f_{N+2}^{1}=0 .
$$

By the assumption that $f^{1}$ is non-degenerate, we obtain

$$
h_{1 j}=h_{2 j}=\cdots=h_{N+2 j} \text {. }
$$

This shows that

$$
f^{1}=f^{2}=\cdots,
$$

which is absurd. We have thus Theorem 4.1.

THEOREM 4.3. Let $\gamma: \boldsymbol{C}^{n} \rightarrow C^{n}$ be a biholomorphic map and $f: \boldsymbol{C}^{n} \rightarrow$ $P^{N}(C)$ a non-degenerate meromorphic map. If there exist hyperplanes $H_{1}, \cdots$, $H_{N+2}$ in general position such that $\nu\left(f, H_{i}\right) \circ \gamma=\nu\left(f, H_{i}\right)(1 \leqq i \leqq N+2)$, then $f \circ \gamma^{j_{0}}=f$ for some positive integer $j_{0}$.

Proof. Consider

$$
\mathscr{F}:=\mathscr{F}\left(H_{1}, \cdots, H_{N+2}, \nu\left(f, H_{1}\right), \cdots, \nu\left(f, H_{N+2}\right)\right) .
$$

Obviously, the assumption implies that $f \circ \gamma^{j} \in \mathscr{F}$ for any positive integer $j$. Since $\# \mathscr{F}<\infty, f \circ \gamma^{j_{1}}=f \circ \gamma^{j_{2}}$ for some $j_{1}, j_{2}$ with $j_{1}<j_{2}$. Then, $f \circ \gamma^{j_{0}}=f$ for $j_{0}:=j_{2}-j_{1}$.

\section{§5. Meromorphic functions of semi-invariant type}

Let $\gamma: C^{n} \rightarrow C^{n}$ be a biholomorphic map and $\Phi$ a family of meromorphic functions on $\boldsymbol{C}^{n}$.

Definition 5.1. We call $\Phi$ a $\gamma$-admissible family if it satisfies the following conditions;

(i) $\Phi$ is a field which includes $C$,

(ii) any $\varphi \in \Phi$ is of order less than one,

(iii) $\Phi$ is $\gamma$-invariant, namely, $\varphi \circ \gamma \in \Phi$ whenever $\varphi \in \Phi$, 
(iv) if $\varphi \circ \gamma^{j}=c \varphi$ for some $\varphi \in \Phi, c \in C$ and a positive integer $j$, then $\varphi \equiv$ const.

ExAmple 5.2. $1^{\circ}$. The field $C$ of all constant functions is obviously a $\gamma$-admissible family for any biholomorphic map $\gamma: C^{n} \rightarrow C^{n}$.

$2^{\circ}$. Let us consider a linear map $\gamma(z)=A z+B$, where $A$ is a regular matrix of order $n$ and $B \in C^{n}$. If there is no pure $(n-1)$-dimensional analytic set $V$ in $C^{n}$ which is of order less than one and $\gamma^{j 0}$-invariant for some positive integer $j_{0}$, then the field $\Phi_{0}$ of all meromorphic functions of order less than one is a $\gamma$-admissible family. In fact, by (2.4) $\Phi_{0}$ is a field and obviously satisfies the conditions (i) $\sim$ (iii). We now suppose that $\varphi \circ \gamma^{j_{0}}=c \varphi$ for some nonconstant $\varphi \in \Phi_{0}, c \in C^{*}$ and a positive integer $j_{0}$. Then, $V:=\left|\nu_{\varphi}^{0}\right| \cup\left|\nu_{\varphi}^{\infty}\right|$ is a $\gamma^{j 0}$-invariant analytic set which is not empty because of (2.9). And, $V$ is of order less than one by (2.6), which contradicts the assumption. Therefore, $\Phi_{0}$ satisfies also the condition (iv).

In the case of $n=1$, the map $\gamma$ defined by $\gamma(z)=z+\omega$ for some $\omega \in C^{*}$ has the above-mentioned property. For, if a discrete set $V$ is $\gamma^{j 0}$-invariant and contains a point $z_{0}$, we have also $z_{0}+j j_{0} \omega \in V$ for $j=1,2, \ldots$. Then, there exists a positive constant $c$ such that

$$
\sharp\{z \in V ;|z| \leqq t\} \geqq c t
$$

for a sufficiently large $t$, and so

$$
N\left(r, \nu_{V}\right) \geqq c r
$$

for a sufficiently large $r$. The set $V$ is not of order less than one.

In the following, $\gamma$ denotes a biholomorphic map of $C^{n}$ onto $C^{n}$ itself and $\Phi$ denotes a $\gamma$-admissible family.

Definition 5.3. A meromorphic function $F(z)$ on $C$ is called to be of $(\gamma, \Phi)$-semi-invariant type if it has a representation

$$
F(z)=\varphi_{1}(z) g_{1}(z)+\cdots+\varphi_{p}(z) g_{p}(z)
$$

with $\varphi_{1}, \cdots, \varphi_{p} \in \Phi$ and meromorphic functions $g_{1}, \cdots, g_{p}$ on $C^{n}$ such that $g_{i} \circ \gamma=c_{i} g_{i}$ for some $c_{i} \in C$.

DeFinition 5.4. A representation (10) is called a reduced representation if it satisfies the conditions;

(i) $F(z) \not \equiv \sum_{i \in I} \varphi_{i} g_{i}$ for any proper subset $I$ of $\{1,2, \cdots, p\}$,

(ii) whenever $c_{i_{1}}=c_{i_{2}}=\cdots=c_{i_{m}},\left\{\varphi_{i_{1}}, \cdots, \varphi_{i_{m}}\right\}$ and $\left\{g_{i_{1}}, \cdots, g_{i_{m}}\right\}$ are 
both linearly independent over $\boldsymbol{C}$.

(5.5) any meromorphic function of $(\gamma, \Phi)$-semi-invariant type has a reduced representation.

Let $F(z)$ have a representation (10) with $\varphi_{i} \in \Phi$ and $g_{i}$ such that $g_{i} \circ \gamma$ $=c_{i} g_{i}$ for some $c_{i} \in C$. Changing indices, we may assume

$$
c_{1}=\cdots=c_{p_{1}}, c_{p_{1}+1}=\cdots=c_{p_{2}}, \cdots, c_{p_{a-1+1}}=\cdots=c_{p_{a}} .
$$

and $c_{p_{\alpha}} \neq c_{p_{\alpha^{\prime}}}$ if $\alpha \neq \alpha^{\prime}$, where $1 \leqq p_{1}<\cdots<p_{a}=p$. For example, for indices $1,2, \cdots, p_{1}$, it may be assumed that $\varphi_{1}, \cdots, \varphi_{r}\left(1 \leqq r \leqq p_{1}\right)$ are linearly independent and

$$
\varphi_{i}=\sum_{j=1}^{r} c_{i j} \varphi_{j} \quad\left(r+1 \leqq i \leqq p_{1}\right)
$$

for some $c_{i j} \in C$. Then, if we set

$$
\tilde{g}_{j}:=g_{j}+\sum_{i=r+1}^{p_{1}} c_{i j} g_{i},
$$

we see $\tilde{g}_{j} \circ \gamma=c_{p_{1}} \tilde{g}_{j}$ and

$$
\sum_{i=1}^{p_{1}} \varphi_{i} g_{i}=\sum_{j=1}^{r} \varphi_{j} \tilde{g}_{j}
$$

Moreover, we may choose indices such that $\tilde{g}_{1}, \cdots, \tilde{g}_{s}$ are linearly independent and

$$
\tilde{\mathbf{g}}_{j}=\sum_{\ell=1}^{s} d_{\ell j} \tilde{\mathbf{g}}_{\ell} \quad(s+1 \leqq j \leqq r)
$$

for some $d_{\ell j} \in C$. We have then

$$
\sum_{i=1}^{p_{1}} \varphi_{i} g_{i}=\sum_{\ell=1}^{s} \tilde{\varphi}_{\ell} \tilde{g}_{\ell}
$$

where $\tilde{\varphi}_{\ell}:=\varphi_{\ell}+\sum_{j=s+1}^{r} d_{\ell j} \varphi_{j} \in \Phi$ and $\tilde{\varphi}_{1}, \cdots, \tilde{\varphi}_{s}$ are linearly independent. By the same reason, $\sum_{i=p_{\alpha-1}+1}^{p_{\alpha}} \varphi_{i} g_{i}$ has a reduced representation for each $\alpha$, whence we conclude (5.5).

THEOREM 5.6. Let $F(z)$ have two reduced representations

$$
F(z)=\sum_{i=1}^{p} \varphi_{i} f_{i}=\sum_{j=1}^{q} \psi_{j} g_{j}
$$

where $\varphi_{i} \in \Phi, \psi_{j} \in \Phi, f_{i} \circ \gamma=c_{i} f_{i}$ and $g_{j} \circ \gamma=d_{j} g_{j}$ for some $c_{i}, d_{j} \in C$. Then, 
$p=q$ and, after a suitable change of indices, we can find a partition of indices

$$
\{1,2, \cdots, p\}=I_{1} \cup I_{2} \cup \cdots \cup I_{a}
$$

satisfying the conditions that, for each $\alpha=1,2, \cdots, a$,

(i) $c_{i}=c_{i^{\prime}}$ and $d_{i}=d_{i^{\prime}}$ if $i, i^{\prime} \in I_{\alpha}$,

(ii) $\sum_{i \in I_{\alpha}} \varphi_{i} f_{i}=\sum_{i \in I_{\alpha}} \psi_{i} g_{i}$,

(iii) there is a regular matrix $C^{\alpha}=\left(c_{i j}^{\alpha} ; i, j \in I_{\alpha}\right)$ such that

$$
g_{j}=\sum_{i \in I_{\alpha}} c_{i j}^{\alpha} f_{i}, \varphi_{i}=\sum_{j \in I_{\alpha}} c_{i j}^{\alpha} \psi_{j} .
$$

For the proof, we need some lemmas.

LEMMA 5.7. Let $\varphi_{1}, \cdots, \varphi_{p}, g_{1}, \cdots, g_{p}$ be non-zero meromorphic functions on $C^{n}$ such that $\varphi_{i} \in \Phi$ and $g_{i} \circ \gamma=c_{i} g_{i}$ for some $c_{i} \in C$. If

$$
\operatorname{det}\left(\left(\varphi_{i} \circ \gamma^{j-1}\right)\left(g_{i} \circ \gamma^{j-1}\right) ; 1 \leqq i, j \leqq p\right) \equiv 0,
$$

then $c_{i_{1}}=c_{i_{2}}=\cdots=c_{i_{m}}$ and $\varphi_{i_{1}}, \varphi_{i_{2}}, \cdots, \varphi_{i_{m}}$ are linearly dependent for some $i_{1}, \cdots, i_{m}$ with $1 \leqq i_{1}<\cdots<i_{m} \leqq p$.

Proof. This is shown by induction on $p$. If $p=2, \varphi_{1} \circ \gamma / \varphi_{2} \circ \gamma=$ $\left(c_{2} / c_{1}\right)\left(\varphi_{1} / \varphi_{2}\right)$ and $\varphi_{1} / \varphi_{2} \in \Phi$ by (11). By Definition 5.1, (iv), $\varphi_{1} / \varphi_{2} \equiv$ const and $c_{1}=c_{2}$, which gives Lemma 5.7. Suppose that Lemma 5.7 is valid in the case $\leqq p-1$. For brevity's sake, we define $f_{i j}:=\left(\varphi_{i} \circ \gamma^{j-1}\right)\left(g_{i} \circ \gamma^{j-1}\right)$. For each $j=p-1, p-2, \ldots$ we subtract the $j$-th column multiplied by $f_{p j+1}$ from the $(j+1)$-th column multiplied by $f_{p j}$ in order. Consequently we obtain

$$
\operatorname{det}\left(f_{p j} f_{i j+1}-f_{p j+1} f_{i j} ; 1 \leqq i, j \leqq p-1\right) \equiv 0
$$

Define

$$
\begin{aligned}
& \tilde{\varphi}_{i}:=c_{i} \varphi_{p}\left(\varphi_{i} \circ \gamma\right)-c_{p} \varphi_{i}\left(\varphi_{p} \circ \gamma\right) \\
& \tilde{g}_{i}:=g_{i} g_{p}
\end{aligned}
$$

for $i=1,2, \cdots, p-1$. Then, $\tilde{\varphi}_{i} \in \Phi, \tilde{g}_{i} \circ \gamma=c_{i} c_{p} \tilde{g}_{i}$ and

$$
\operatorname{det}\left(\left(\tilde{\varphi}_{i} \circ \gamma^{j-1}\right)\left(\tilde{g}_{i} \circ \gamma^{j-1}\right) ; 1 \leqq i, j \leqq p-1\right) \equiv 0 .
$$

If $\tilde{\varphi}_{i} \tilde{g}_{i}=f_{p 1} f_{i 2}-f_{p 2} f_{i 1} \equiv 0$, we have easily $c_{i}=c_{p}, \varphi_{i} / \varphi_{p} \equiv$ const and so the conclusion of Lemma 5.7. We may assume $\tilde{\varphi}_{i} \tilde{g}_{i} \equiv \equiv 0$ for any $i$. We now apply the induction hypothesis to functions $\tilde{\varphi}_{i}, \tilde{g}_{i}$. There are indices $i_{1}, \cdots$, 
$i_{m-1}$ with $1 \leqq i_{1}<\cdots<i_{m-1} \leqq p-1$ such that $c_{i_{1}}=\cdots=c_{i_{m-1}}$ and

$$
\sum_{\ell} a_{\ell} \tilde{\varphi}_{i_{\ell}}=\varphi_{p}\left(\sum_{\ell} a_{\ell} c_{i_{\ell}}\left(\varphi_{i_{\ell}} \circ \gamma\right)\right)-c_{p}\left(\varphi_{p} \circ \gamma\right)\left(\sum_{\ell} a_{\ell} \varphi_{i_{\ell}}\right)=0
$$

for some non-zero vector $\left(a_{1}, \cdots, a_{m-1}\right)$. This implies that a function $\psi:=$ $\sum_{\ell} a_{\ell} \varphi_{i_{\ell}} / \varphi_{p}$ in $\Phi$ satisfies $\psi \circ \gamma=\left(c_{p} / c_{i_{1}}\right) \psi$, where we may assume $\psi \not \equiv 0$. By Definition 5.1, (iv), $\psi \equiv$ const and $c_{i_{1}}=c_{p}$. Consequently, $c_{i_{1}}=\cdots=c_{i_{m-1}}$ $=c_{p}$ and $\varphi_{i_{1}}, \cdots, \varphi_{i_{m-1}}, \varphi_{p}$ are linearly dependent. The proof is completed.

LemMa 5.8. Let $\varphi_{1}, \cdots, \varphi_{p}, g_{1}, \cdots, g_{p}$ be functions as in Lemma 5.7 and assume that

$$
\varphi_{1} g_{1}+\varphi_{2} g_{2}+\cdots+\varphi_{p} g_{p} \equiv 0 \text {. }
$$

Consider the partition of indices

$$
\{1,2, \cdots, p\}=I_{1} \cup I_{2} \cup \cdots \cup I_{a}
$$

such that, for any $i \in I_{\alpha}$ and $i^{\prime} \in I_{\alpha^{\prime}}, c_{i}=c_{i^{\prime}}$ if $\alpha=\alpha^{\prime}$, and $c_{i} \neq c_{i^{\prime}}$ if $\alpha \neq \alpha^{\prime}$. Then, for any $\alpha$,

(i) $\sum_{i \in I_{\alpha}} \varphi_{i} g_{i} \equiv 0$,

(ii) $\left\{\varphi_{i} ; i \in I_{\alpha}\right\}$ are linearly dependent.

Proof. By (13), we have

$$
\left(\varphi_{1} \circ \gamma^{j-1}\right)\left(g_{1} \circ \gamma^{j-1}\right)+\cdots+\left(\varphi_{p} \circ \gamma^{j-1}\right)\left(g_{p} \circ \gamma^{j-1}\right) \equiv 0
$$

for $j=1,2, \cdots, p$. Therefore,

$$
\operatorname{det}\left(\left(\varphi_{i} \circ \gamma^{j-1}\right)\left(g_{i} \circ \gamma^{j-1}\right) ; 1 \leqq i, j \leqq p\right) \equiv 0 .
$$

By Lemma 5.7, $\left\{\varphi_{i} ; i \in I_{\alpha}\right\}$ are linearly dependent for some $\alpha$. This shows that (ii) is a consequence of (i). To prove (i), it suffices to get an absurd conclusion under the assumption that

$$
F_{\alpha}:=\sum_{i \in I_{\alpha}} \varphi_{i} g_{i} \not \equiv 0
$$

for any $\alpha$. Take a reduced representation

$$
F_{\alpha}(z)=\sum_{i \in \dot{I}_{\alpha}} \tilde{\varphi}_{i}(z) \tilde{g}_{i}(z)
$$

where $\tilde{I}_{\alpha} \subseteq I_{\alpha}, \tilde{\varphi}_{i} \in \Phi$ and $\tilde{g}_{i} \circ \gamma=c_{i_{\alpha}} \tilde{g}_{i}$ for some $i_{\alpha} \in I_{\alpha}$. Then, the identity

$$
\sum_{\alpha=1}^{a} \sum_{i \in I_{\alpha}} \tilde{\varphi}_{i} \tilde{g}_{i}=0
$$


contradicts Lemma 5.7. We have thus Lemma 5.8 .

LeMma 5.9. Let $\varphi_{1}, \cdots, \varphi_{p} \in \Phi$ and $g_{1}, \cdots, g_{p}$ be meromorphic functions on $C^{n}$ such that $g_{i} \circ \gamma=c_{i} g_{i}$ for some $c_{i} \in C$ and

$$
\varphi_{1} g_{1}+\varphi_{2} g_{2}+\cdots+\varphi_{p} g_{p} \equiv 0 \text {. }
$$

If $\varphi_{1}, \cdots, \varphi_{p}$ are linearly independent, then $g_{1} \equiv \cdots \equiv g_{p} \equiv 0$.

Proof. If $g_{i_{0}} \neq \equiv$ for some $i_{0},\left\{\varphi_{i} ; c_{i}=c_{i_{0}}\right\}$ are linearly dependent by Lemma 5.8. This contradicts the assumption. We have thus the conclusion of Lemma 5.9 .

Proof of Theorem 5.6. Take the partitions of indices

$$
\begin{aligned}
& \{1,2, \cdots, p\}=I_{1} \cup I_{2} \cup \cdots \cup I_{a} \\
& \{1,2, \cdots, q\}=J_{1} \cup J_{2} \cup \cdots \cup J_{b}
\end{aligned}
$$

such that, for $i \in I_{\alpha}, i^{\prime} \in I_{\alpha^{\prime}}, j \in J_{\beta}, j^{\prime} \in J_{\beta^{\prime}}$, we have $c_{i}=c_{i^{\prime}}, d_{j}=d_{j^{\prime}}$ if $\alpha=\alpha^{\prime}$, $\beta=\beta^{\prime}$, and $c_{i} \neq c_{i^{\prime}}, d_{j} \neq d_{j^{\prime}}$ if $\alpha \neq \alpha^{\prime}, \beta \neq \beta^{\prime}$. Define

$$
\begin{aligned}
& F_{\alpha}:=\sum_{i \in I_{\alpha}} \varphi_{i} f_{i}, \\
& G_{\beta}:=\sum_{j \in I_{\beta}} \psi_{j} g_{j},
\end{aligned}
$$

which do not vanish by Definition 5.4, (i). Apply Lemma 5.8 to the identity

$$
\sum_{i=1}^{p} \varphi_{i} f_{i}-\sum_{j=1}^{q} \psi_{j} g_{j} \equiv 0 \text {. }
$$

We see easily $a=b$ and $F_{\alpha} \equiv G_{\alpha}(1 \leqq \alpha \leqq a)$ after a suitable change of indices. This gives (i) and (ii) of Theorem 5.6.

To prove (iii), we may assume $a=b=1$ and so $c_{1}=\cdots=c_{p}=d_{1}$ $=\cdots=d_{q}$. Since $\psi_{1}, \cdots, \psi_{q}$ are linearly independent, we can choose indices such that $\psi_{1}, \cdots, \psi_{q}, \varphi_{1}, \cdots, \varphi_{r}$ are linearly independent and

$$
\varphi_{i}=\sum_{j=1}^{q} c_{i j} \psi_{j}+\sum_{j=1}^{r} d_{i j} \varphi_{j} \quad(\mathrm{r}+1 \leqq i \leqq p)
$$

where $0 \leqq r \leqq p$ and $c_{i j}, d_{i j} \in C$. Then

$$
\sum_{j=1}^{q} \psi_{j}\left(g_{j}-\sum_{i=r+1}^{p} c_{i j} f_{i}\right)-\sum_{j=1}^{r} \varphi_{j}\left(f_{j}+\sum_{i=r+1}^{p} d_{i j} f_{i}\right)=0 .
$$

It follows from Lemma 5.9 that

$$
g_{j}=\sum_{i=r+1}^{p} c_{i j} f_{i} \quad(1 \leqq j \leqq q),
$$




$$
f_{j}+\sum_{i=r+1}^{p} d_{i j} f_{i}=0 \quad(1 \leqq j \leqq r)
$$

We note here the case $r \geqq 1$ is impossible because $f_{1}, \cdots, f_{p}$ are linearly independent. Therefore, (14) becomes

$$
\varphi_{i}=\sum_{j=1}^{q} c_{i j} \psi_{j} .
$$

The similar argument is available if we exchange the roles of $f_{i}$ 's and $g_{j}$ 's. We can conclude that $p=q$ and $C=\left(c_{i j}\right)$ is a regular matrix.

\section{§6. Meromorphic functions with $\gamma$-invariant zeros and poles}

In this section, $\gamma$ denotes a biholomorphic map of $\boldsymbol{C}^{n}$ onto $\boldsymbol{C}^{n}$ itself and $\Phi$ denotes a $\gamma$-admissible family. For non-zero meromorphic functions $g_{1}$ and $g_{2}$ on $C^{n}$, we mean by notation $g_{1} \underset{\gamma}{\sim} g_{2}$ that $g_{1} / g_{2}$ is $\gamma^{j 0^{0} \text {-invariant, }}$ namely, $g_{1} \circ \gamma^{j_{0}} / g_{2} \circ \gamma^{j_{0}}=g_{1} / g_{2}$ for some positive integer $j_{0}$.

TheOREM 6.1. Let $\varphi_{1}, \cdots, \varphi_{p} \in \Phi$ and $g_{1}, \cdots, g_{p}$ be non-zero meromorphic functions with $\nu_{g_{i}} \circ \gamma=\nu_{g_{i}}$. If

$$
\varphi_{1} g_{1}+\varphi_{2} g_{2}+\cdots+\varphi_{p} g_{p} \equiv 0
$$

and $\sum_{i \in I} \varphi_{i} g_{i} \not \equiv 0$ for any proper subset $I$ of $\{1,2, \cdots, p\}$, then

$$
g_{1} \underset{\gamma}{\sim} g_{2} \underset{\gamma}{\sim} \cdots \underset{\gamma}{\sim} g_{p} .
$$

For the proof, we give

Lemma 6.2. Let $\varphi_{1}, \cdots, \varphi_{p} \in \Phi$ and $g_{1}, \cdots, g_{p}$ be meromorphic functions such that $\varphi_{i} \not \equiv 0, g_{i} \not \equiv 0$ and $\nu_{g_{i}} \circ \gamma=\nu_{g_{i}}$. If

$$
\operatorname{det}\left(\left(\varphi_{i} \circ \gamma^{j-1}\right)\left(g_{i} \circ \gamma^{j-1}\right) ; i=1,2, \cdots, p, j=j_{1}, \cdots, j_{p}\right) \equiv 0
$$

for any $j_{1}, j_{2}, \cdots, j_{p}$, then

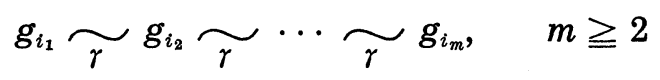

and $\varphi_{i_{1}}, \cdots, \varphi_{i_{m}}$ are linearly dependent over $C$ for some indices $i_{1}, \cdots, i_{m}$ with $1 \leqq i_{1}<i_{2}<\cdots<i_{m} \leqq p$.

Proof. We prove this by induction on $p$. If $p=2$, we have

$$
\frac{\varphi_{1}}{\varphi_{2}} \frac{\varphi_{2} \circ \gamma}{\varphi_{1} \circ \gamma}=\frac{g_{1} \circ \gamma}{g_{1}} \frac{g_{2}}{g_{2} \circ \gamma} .
$$


This is reduced to a constant $c$ because the left side is in $\Phi$ and the right side is in $H^{*}$ by the assumption. Therefore,

$$
\frac{\varphi_{1} \circ \gamma}{\varphi_{2} \circ \gamma}=c \frac{\varphi_{1}}{\varphi_{2}}
$$

And, $\varphi_{1} / \varphi_{2} \equiv$ const and $c=1$ by virtue of Definition 5.1, (iv). This implies. the $\gamma$-invariance of $g_{1} / g_{2}$.

Suppose that Lemma 6.2 is true in the case $\leqq p-1$. Changing indices, we may assume that

( $\alpha)$ if $r+1 \leqq i_{1}<i_{2} \leqq p$, then $g_{i_{1}} \circ \gamma^{j} / g_{i_{2}} \circ \gamma^{j}=c g_{i_{1}} / g_{i_{2}}$ for some positive integer $j$ and a constant $c$,

( $\beta)$ if $1 \leqq i_{1} \leqq r$ and $r+1 \leqq i_{2} \leqq p$, then there is no constant $c$ with such a property for any $j$.

Moreover, replacing $\gamma^{j}$ by $\gamma$, we may take $j=1$ in $(\alpha)$. On the other hand, (16) remains valid if we divide the $j$-th column of the matrix

$$
\left(\left(\varphi_{i} \circ \gamma^{j-1}\right)\left(g_{i} \circ \gamma^{j-1}\right) ; i=1,2, \cdots, p, j=1,2, \cdots\right)
$$

by $g_{p} \circ \gamma^{j-1}$. Replacing $g_{i} / g_{p}$ by $g_{i}$, we may assume that $g_{p} \equiv 1$ and so $g_{r+1}, \cdots, g_{p}$ satisfies $g_{i} \circ \gamma=c_{i} g_{i}$ for some $c_{i}$. Define

$$
h_{i j}:=\frac{g_{i} \circ \gamma^{j-1}}{g_{i}}
$$

for any $i=1,2, \cdots, p$ and $j=1,2, \cdots$. Then, $h_{i j} \equiv$ const for any $j$ if $r+1$ $\leqq i \leqq p$. Moreover, $h_{i j_{1}} / h_{i j_{2}} \neq$ const if $1 \leqq i \leqq r$ and $j_{1}<j_{2}$. For, if not, $g_{i} \circ \gamma^{j_{2}-j_{1}} / g_{i} \equiv$ const, which contradicts the above condition $(\beta)$. Divide the $i$-th row of (16) by $g_{i}$. We have then

$$
\operatorname{det}\left(\left(\varphi_{i} \circ \gamma^{j-1}\right) h_{i j} ; i=1,2, \cdots, p, j=j_{1}, \cdots, j_{p}\right) \equiv 0
$$

for any $j_{1}, \cdots, j_{p}$.

We now assume that the conclusion of Lemma 6.2 is false, namely, $\varphi_{i_{1}}, \cdots, \varphi_{i_{m}}$ are linearly independent whenever

$$
g_{i_{1}} \widetilde{r} g_{i_{2}} \widetilde{\gamma} \cdots \underset{r}{\sim} g_{i_{m}} .
$$

Give a positive integer $j$ arbitrarily and define

$$
j_{r+1}^{*}:=j, j_{r+2}^{*}:=2 j, \cdots, j_{p}^{*}:=(p-r) j .
$$

And, apply Lemma 5.7 to $\gamma^{j}, \varphi_{i} \circ \gamma^{j}$ and $g_{i} \circ \gamma^{j}(r+1 \leqq i \leqq p)$ instead of $\gamma$, $\varphi_{i}$ and $g_{i}$ respectively. As its consequence, we see 


$$
\operatorname{det}\left(\left(\varphi_{i} \circ \gamma^{k j}\right)\left(g_{i} \circ \gamma^{k j}\right) ; i=r+1, \cdots, p, k=1, \cdots, p-r\right) \not \equiv 0,
$$

whence

$$
\operatorname{det}\left(\left(\varphi_{i} \circ \gamma^{j-1}\right) h_{i j} ; i=r+1, \cdots, p, j=j_{r+1}^{*}, \cdots, j_{p}^{*}\right) \not \equiv 0 .
$$

This shows that a matrix $\mathscr{M}=\left(\left(\varphi_{i} \circ \gamma^{j-1}\right) h_{i j}\right)$ satisfies all assumptions of Lemma 3.2. We can conclude

$$
\operatorname{det}\left(\left(\varphi_{i} \circ \gamma^{j-1}\right)\left(g_{i} \circ \gamma^{j-1}\right) ; i=1,2, \cdots, r, j=j_{1}, \cdots, j_{r}\right) \equiv 0
$$

for any $j_{1}, \cdots, j_{r}$. On the other hand, by the assumption the conclusion of Lemma 5.7 does not occur. This is a contradiction. We have thus Lemma 6.2.

Proof of Theorem 6.1. According to (15), we have

$$
\left(\varphi_{1} \circ \gamma^{j-1}\right)\left(g_{1} \circ \gamma^{j-1}\right)+\cdots+\left(\varphi_{p} \circ \gamma^{j-1}\right)\left(g_{p} \circ \gamma^{j-1}\right) \equiv 0
$$

for any $j=1,2, \cdots$. Therefore,

$$
\operatorname{det}\left(\left(\varphi_{i} \circ \gamma^{j-1}\right)\left(g_{i} \circ \gamma^{j-1}\right) ; i=1, \cdots, p, j=j_{1}, \cdots, j_{p}\right) \equiv 0
$$

for any $j_{1}, \cdots, j_{p}$. If $p=2$, the desired conclusion is a direct result of Lemma 6.2. Now, suppose that Theorem 6.1 is true in the case $\leqq p-1$ and false in the case $p$. Changing indices, we may assume

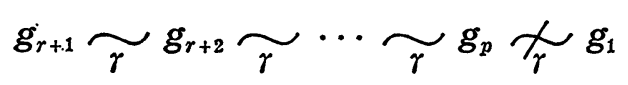

and $\varphi_{r+1}, \cdots, \varphi_{p}$ are linearly dependent over $C$ by the help of Lemma 6.2, where $1 \leqq r<p$. Replacing $g_{i} g_{p}^{-1}$ by $g_{i}$ and $\gamma^{j}$ by $\gamma$ for a suitable positive integer $j$, each $g_{i}$ with $r+1 \leqq i \leqq p$ may be assumed to be $\gamma$-invariant. Moreover, we may write

$$
\varphi_{p}=c_{r+1} \varphi_{r+1}+\cdots+c_{p-1} \varphi_{p-1}
$$

with some constants $c_{r+1}, \cdots, c_{p-1}$. Define

$$
\begin{aligned}
& \tilde{g}_{i}:=g_{i} \quad(1 \leqq i \leqq r) \\
& \tilde{g}_{i}:=g_{i}+c_{i} g_{p} \quad(r+1 \leqq i \leqq p-1) .
\end{aligned}
$$

Then, $\tilde{g}_{r+1}, \cdots, \tilde{g}_{p-1}$ are $\gamma$-invariant and (15) is rewritten as

$$
\sum_{i=1}^{p-1} \varphi_{i} \tilde{g}_{i}=0 \text {. }
$$

Take a subset $I$ of $\{1,2, \cdots, p-1\}$ which is minimal among subsets with the property that $1 \in I$ and 


$$
\sum_{i \in I} \varphi_{i} \tilde{g}_{i} \equiv 0 .
$$

By the assumption, $I \nsubseteq\{1,2, \cdots, r\}$ and so $I$ contains some $i_{0}$ in $\{r+1, \cdots$, $p-1\}$. Since $\sharp I \leqq p-1$, we can conclude form (17)

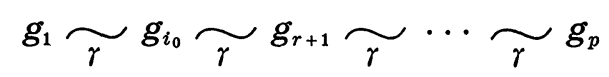

by the induction hypothesis. This is a contradiction. Theorem 6.1 is true in the case $p$ too. Consequently, we have Theorem 6.1.

Corollary 6.3. Let $\varphi_{1}, \cdots, \varphi_{p}$ be non-zero functions in $\Phi$ and $g_{1}, \cdots, g_{p}$ non-zero meromorphic functions on $C^{n}$ with $\nu_{g_{i}} \circ \gamma=\nu_{g_{i}}$ satisfying

$$
\varphi_{1} g_{1}+\varphi_{2} g_{2}+\cdots+\varphi_{p} g_{p} \equiv 0 .
$$

Then, there exists a partition of indices

$$
\{1,2, \cdots, p\}=I_{1} \cup I_{2} \cup \cdots \cup I_{a}
$$

such that, for any $\alpha$,

$$
\sum_{i \in I_{\alpha}} \varphi_{i} g_{i} \equiv 0
$$

and $g_{i} \underset{\gamma}{\sim} g_{i^{\prime}}$ if $i, i^{\prime} \in I_{\alpha}$.

Proof. It suffices to take a partition

$$
\{1,2, \cdots, p\}=I_{1} \cup \cdots \cup I_{a}
$$

such that, for any $\alpha, \sum_{i \in I_{\alpha}} \varphi_{i} g_{i} \equiv 0$ and $\sum_{i \in I_{\alpha}^{\prime}} \varphi_{i} g_{i} \not \equiv 0$ whenever $I_{\alpha}^{\prime} \subsetneq I_{\alpha}$. By Theorem 6.1, we see easily $g_{i} \underset{\gamma}{\sim} g_{i^{\prime}}$ for any $i, i^{\prime} \in I_{\alpha}$.

\section{§7. Generalizations of Urabe-Yang's results}

In this section, we restrict ourselves to the study of meromorphic functions on $C$. As in $\S \S 2$ and 3 we denote by $\Phi_{0}$ the set of all meromorphic functions of order less than one. We consider a biholomorphic functions of order less than one. We consider a biholomorphic map $\gamma_{\omega}: C \rightarrow C$ defined by $\gamma_{\omega}(z)=z+\omega$ for a constant $\omega \in C^{*}$.

We first give the following generalization of a result in [12].

Let us consider meromorphic functions

$$
\begin{aligned}
& F:=\varphi_{0}+\sum_{i=1}^{p} \varphi_{i} f_{i} \\
& G:=\psi_{0}+\sum_{j=1}^{q} \psi_{j} g_{j}
\end{aligned}
$$


satisfying the conditions:

(i) $\varphi_{0}, \varphi_{1}, \cdots, \varphi_{p}, \psi_{0}, \psi_{1}, \cdots, \psi_{q} \in \Phi_{0}$ and $f_{1}, \cdots, f_{p}, g_{1}, \cdots, g_{q}$ are nonzero holomorphic functions on $C$ such that $f_{i} \circ \gamma_{\omega_{1}}=f_{i}$ and $g_{j} \circ \gamma_{\omega_{2}}=g_{j}$ for some $\omega_{1}, \omega_{2} \in C^{*}$,

(ii) (18) are both reduced representations when they are regarded as meromorphic functions of $\left(\gamma_{\omega_{1}}, \Phi_{0}\right)$ - and $\left(\gamma_{\omega_{2}}, \Phi_{0}\right)$-semi-invariant type respectively,

(iii) $\psi_{0}$ does not belong to the set $\left\{\varphi_{i}, \psi_{1}, \cdots, \psi_{q}\right\}_{c}$ of all linear combinations of $\varphi_{i}, \psi_{1}, \cdots, \psi_{q}$ with constant coefficients for any $i=1,2, \cdots, p$,

(iv) $\min \left\{\nu_{\varphi_{0}}, \nu_{\varphi_{1}}, \cdots, \nu_{\varphi_{p}}\right\}=\min \left\{\nu_{\psi_{0}}, \nu_{\psi_{1}}, \cdots, \nu_{\psi_{q}}\right\}$.

THEOREM 7.1. If $\nu_{F}-\nu_{G}$ is of order less than one, then $\omega_{1} / \omega_{2}$ is $a$ rational number and $F(z)=c G(z)$ for some $c \in C^{*}$.

Remark. In the special case where $p=q=1$ and $\psi_{1}=\varphi_{1}=1$, Theorem 7.1 is Theorem 1 in [12].

For the proof of Theorem 7.1, we need

LEMMA 7.2. Let $\varphi_{1}, \cdots, \varphi_{p}, \psi_{1}, \cdots, \psi_{q} \in \Phi_{0}$ and $f_{1}, \cdots, f_{p}, g_{1}, \cdots, g_{q}$ be holomorphic functions on $C$ such that $f_{i} \circ \gamma_{\omega_{1}}=f_{i}$ and $g_{j} \circ \gamma_{\omega_{2}}=g_{j}$ for some $\omega_{1}, \omega_{2} \in C^{*}$. If

$$
\sum_{i=1}^{p} \varphi_{i} f_{i}=\sum_{j=1}^{q} \psi_{j} g_{j}=: F(z)
$$

and $F(z)$ is not of order less than one, then $\omega_{1} / \omega_{2}$ is a rational number.

Proof. It may be assumed that $\sum_{i=1}^{p} \varphi_{i} f_{i}$ and $\sum_{j=1}^{q} \psi_{j} g_{j}$ are both reduced representations of $F(z)$ when they are regarded as meromorphic functions of $\left(\gamma_{\omega_{1}}, \Phi_{0}\right)$ - and $\left(\gamma_{\omega_{2}}, \Phi_{0}\right)$-semi-invariant type.

We first study the case $q=1$. Without loss of generality, we may assume $\psi_{1} \equiv 1$ and so $F \circ \gamma_{\omega_{2}}=F$. We have then

$$
\sum_{i=1}^{p}\left(\varphi_{i} \circ \gamma_{\omega_{2}}\right)\left(f_{i} \circ \gamma_{\omega_{2}}\right)=\sum_{i=1}^{p} \varphi_{i} f_{i}
$$

We note that $f_{i}$ and $f_{i} \circ \gamma_{\omega_{2}}$ are $\gamma_{\omega_{1}}$-invariant. We can regard both sides of (19) as reduced representations of a meromorphic function of $\left(\gamma_{\omega_{1}}, \Phi_{0}\right)$ semi-invariant type. By the help of Theorem 5.6, (iii), we can find a regular matrix $C=\left(c_{i j}\right)$ such that 


$$
\begin{aligned}
& \varphi_{i}\left(z+\omega_{2}\right)=\sum_{j=1}^{p} c_{i j} \varphi_{j}(z) \\
& f_{j}(z)=\sum_{i=1}^{p} c_{i j} f_{i}\left(z+\omega_{2}\right) .
\end{aligned}
$$

Then, by the classical theorem of Jordan, if we take a regular linear transformation

$$
\tilde{\varphi}_{k}=\sum_{\ell=1}^{p} r_{k \ell} \varphi_{\ell}
$$

suitably, (20) is reduced to the relations

$$
\tilde{\varphi}_{i}\left(z+\omega_{2}\right)=\sum_{j=1}^{p} d_{i j} \tilde{\varphi}_{j}
$$

such that $\lambda_{i}:=d_{i i} \neq 0, \varepsilon_{i}:=d_{i i+1}$ is equal either to 0 or to 1 , and $d_{i j}=0$ if $i \geqq j+1$ or $j>i+1$, where $\lambda_{i}=\lambda_{i+1}$ if $\varepsilon_{i}=1$. Particularly, we see $\tilde{\varphi}_{p}\left(z+\omega_{2}\right)=\lambda_{p} \tilde{\varphi}_{p}$. As is shown in Example 5.2, $2^{\circ}$, it follows that $\tilde{\varphi}_{p} \equiv$ const and $\lambda_{p}=1$. If $\varepsilon_{i}=0$ for some $i(1 \leqq i \leqq p-1)$, we see also $\tilde{\varphi}_{i}\left(z+\omega_{2}\right)=$ $\lambda_{i} \tilde{\varphi}_{i}(z)$ and hence $\tilde{\varphi}_{i} \equiv$ const. This is a contradiction because $\tilde{\varphi}_{1}, \cdots, \tilde{\varphi}_{p}$ are linearly independent. Therefore, $\varepsilon_{1}=\cdots=\varepsilon_{p-1}=1$ and so $\lambda_{1}=\lambda_{2}=\cdots$ $=\lambda_{p}=1$. Define

$$
{ }_{\ell}(z)=\sum_{k=1}^{p} r_{k \ell} f_{k}(z)
$$

Then,

$$
\tilde{f}_{j}(z)=\sum_{i=1}^{p} d_{i j} \tilde{f}_{i}\left(z+\omega_{2}\right) .
$$

In particular, $\tilde{f}_{1}(z)=\tilde{f}_{1}\left(z+\omega_{2}\right)$ and $\tilde{f}_{2}(z)=\tilde{f}_{2}\left(z+\omega_{2}\right)+\tilde{f}_{1}\left(z+\omega_{2}\right)$. Now, assume that $\omega_{1} / \omega_{2}$ is not a rational number. Since $\tilde{f}_{1}(z)$ is a periodic holomorphic function with period $\omega_{1}$ and simultaneously $\omega_{2}$, it must be a constant. Then,

$$
\tilde{f}_{2}^{\prime}(z)=\tilde{f}_{2}^{\prime}\left(z+\omega_{2}\right) \text {. }
$$

$\tilde{f}_{2}^{\prime}$ is also periodic with period $\omega_{1}$ and $\omega_{2}$. Hence, $\tilde{f}_{2}^{\prime}(z) \equiv$ const $=: c$. We can write

$$
\tilde{f}_{2}(z)=c z+d,
$$

where $d \in C$. On the other hand, $\tilde{f}_{2}\left(z+\omega_{1}\right)=\tilde{f}_{2}(z)$. We conclude $c=0$ and so $\tilde{f}_{2}(z) \equiv$ const. This is absurd because $f_{1}, \cdots, f_{p}$ are linearly independent. Consequently, $\omega_{1} / \omega_{2}$ is a rational number. 
Now, we shall prove Lemma 7.2 in the general case. By the assumption,

$$
\sum_{i=1}^{p} \varphi_{i}\left(z+k \omega_{2}\right) f_{i}\left(z+k \omega_{2}\right)=\sum_{j=1}^{q} \psi_{j}\left(z+k \omega_{2}\right) g_{j}(z)
$$

for any $k=0,1,2, \ldots$. Since $\psi_{1}, \cdots, \psi_{q}$ are assumed to be linearly independent, we have

$$
\operatorname{det}\left(\psi_{j}\left(z+(k-1) \omega_{2}\right) ; 1 \leqq j, k \leqq q\right) \not \equiv 0
$$

as a result of Lemma 5.7. Choosing $\chi_{i k}^{j} \in \Phi_{0}$ suitably, we get

$$
g_{j}(z)=\sum_{i k} \chi_{i k}^{j}(z) f_{i}\left(z+(k-1) \omega_{2}\right) .
$$

Since $g_{j}(z)$ and $f_{i}\left(z+(k-1) \omega_{2}\right)$ are periodic with period $\omega_{2}$ and $\omega_{1}$ respectively, by applying Lemma 7.2 with $q=1$, we conclude that $\omega_{1} / \omega_{2}$ is a rational number.

Proof of Theorem 7.1. By the assumption, we can write

$$
F(z)=h(z) \varphi(z) G(z)
$$

with $h \in \boldsymbol{H}^{*}$ and $\varphi \in \Phi_{0}$. Substituting $z+k \omega_{1}$ for $z$ in this identity, for each $k=0,1, \cdots, p$ we have

$$
\begin{aligned}
\varphi_{0}(z & \left.+k \omega_{1}\right)+\sum_{i=1}^{p} \varphi_{i}\left(z+k \omega_{1}\right) f_{i}(z) \\
& =h\left(z+k \omega_{1}\right) \varphi\left(z+k \omega_{1}\right)\left(\psi_{0}\left(z+k \omega_{1}\right)+\sum_{j=1}^{q} \psi_{j}\left(z+k \omega_{1}\right) g_{j}\left(z+k \omega_{1}\right)\right),
\end{aligned}
$$

both sides of which we denote by $\chi_{k}(z)$. Eliminating $f_{1}, \cdots, f_{p}$, we obtain

$$
\begin{aligned}
\Phi(z): & =\operatorname{det}\left(\varphi_{0}\left(z+k \omega_{1}\right), \varphi_{1}\left(z+k \omega_{1}\right), \cdots, \varphi_{p}\left(z+k \omega_{1}\right) ; 0 \leqq k \leqq p\right) \\
& =\operatorname{det}\left(\chi_{k}(z), \varphi_{1}\left(z+k \omega_{1}\right), \cdots, \varphi_{p}\left(z+k \omega_{1}\right) ; 0 \leqq k \leqq p\right),
\end{aligned}
$$

the right side of which we may rewrite

$$
\Phi(z)=\sum_{0 \leqq k \leqq p, 0 \leqq \ell \leqq q} \tilde{\varphi}_{k l}(z) h\left(z+k \omega_{1}\right) g_{\ell}\left(z+k \omega_{1}\right)
$$

where $g_{0} \equiv 1$ and $\tilde{\varphi}_{k \ell} \in \Phi_{0}$. Since $\varphi_{0}, \varphi_{1}, \cdots, \varphi_{p}$ are linearly independent, $\Phi(z)$ $\not \equiv 0$ by Lemma 5.7. Then, as is easily seen by Corollary 6.3 , we can find

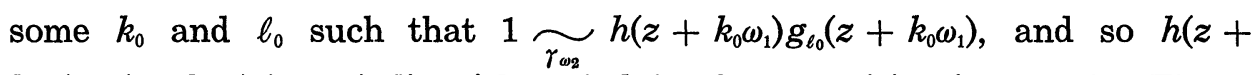
$\left.k_{0} \omega_{1}\right) g_{\ell_{0}}\left(z+k_{0} \omega_{1}\right)$ is periodic with period $j_{0} \omega_{2}$ for a positive integer $j_{0}$. Therefore, $h(z)$ itself is periodic with period $j_{0} \omega_{2}$. In view of (21) and Lemma 
7.2, we can conclude that $\omega_{1} / \omega_{2}$ is a rational number. Then, $f_{i}, g_{j}$ and $h$ are all periodic with period $\omega:=k_{1} \omega_{1}=k_{2} \omega_{2}$ for some non-zero integers $k_{1}$, $k_{2}$. We may regard both sides of the identity

$$
\varphi_{0}+\sum_{i=1}^{p} \varphi_{i} f_{i}=\varphi \psi_{0} h+\sum_{j=1}^{q} \varphi \psi_{j} h g_{j}
$$

as two reduced representations of a meromorphic function $F(z)$ of $\left(\gamma_{\omega}, \Phi_{0}\right)$ semi-invariant type. According to Theorem 5.6, (iii), $p=q$ and there is a regular matrix $C=\left(c_{i j}\right)$ such that

$$
\begin{array}{ll}
\varphi_{i}=\sum_{j=0}^{p} c_{i j} \varphi \psi_{j} & (0 \leqq i \leqq p) \\
g_{j} h=\sum_{i=0}^{p} c_{i j} f_{i} & (0 \leqq j \leqq p),
\end{array}
$$

where $f_{0} \equiv g_{0} \equiv 1$.

We now take a function $\chi \in \Phi_{0}$ such that

$$
\nu_{x}=\min \left(\nu_{\varphi_{0}}, \cdots, \nu_{\varphi_{p}}\right)=\min \left(\nu_{\psi_{0}}, \cdots, \nu_{\psi_{q}}\right)
$$

by the use of (2.7). Changing $\chi \varphi_{i}$ and $\chi \psi_{j}$ by $\varphi_{i}$ and $\psi_{j}$ respectively, we may assume that $\varphi_{0}, \cdots, \varphi_{p}, \psi_{0}, \cdots, \psi_{q}$ are all holomorphic and

$$
\left\{\varphi_{0}=\cdots=\varphi_{p}=0\right\}=\left\{\psi_{0}=\cdots=\psi_{q}=0\right\}=\phi .
$$

We next write $\varphi=\beta / \alpha$ with holomorphic functions $\alpha, \beta \in \Phi_{0}$ which have no common zero. Then, (22) becomes

$$
\alpha \varphi_{i}=\sum_{j} c_{i j} \beta \psi_{j} .
$$

If $\beta \not \equiv$ const, $\beta$ has a zero $z_{0} \in C$ because of (2.7). Then, $\alpha\left(z_{0}\right) \varphi_{i}\left(z_{0}\right)=0$ for any $i$ and so $\alpha\left(z_{0}\right)=0$ by (24), which is absurd. We conclude $\beta \equiv$ const. Similarly, we see $\alpha \equiv$ const. We may assume $\varphi \equiv 1$. In (22), if $c_{i 0} \neq 0$ for some $i$ with $1 \leqq i \leqq p$, then $\psi_{0} \in\left\{\varphi_{i}, \psi_{1}, \cdots, \psi_{p}\right\}_{c}$ which contradicts the assumption (iii). So, $c_{i 0}=0$ for $i=1,2, \cdots, p$. We conclude from (23)

$$
h=g_{0} h=c_{00} \equiv \text { const . }
$$

This shows Theorem 7.1.

Let us consider two entire functions

$$
\begin{aligned}
& F=\sum_{i=1}^{p} \varphi_{i} f_{i} \\
& G=\sum_{j=1}^{q} \psi_{j} g_{j},
\end{aligned}
$$


where $\varphi_{1}, \cdots, \varphi_{p}, \psi_{1}, \cdots, \psi_{q}$ are entire functions of order less than one, and $f_{1}, \cdots, f_{p}$ and $g_{1}, \cdots, g_{q}$ are periodic entire functions with period $\omega_{1}$ and $\omega_{2}$ respectively.

Corollary 7.3. Assume that $\left\{z, \varphi_{1}, \cdots, \varphi_{p}\right\},\left\{z, \psi_{1}, \cdots, \psi_{q}\right\},\left\{1, f_{1}, \cdots, f_{p}\right\}$ and $\left\{1, g_{1}, \cdots, g_{q}\right\}$ are all linearly independent, and $z \notin\left\{\varphi_{i}, \psi_{1}, \cdots, \psi_{q}\right\}_{c}, z \notin$ $\left\{\psi_{j}, \varphi_{1}, \cdots, \varphi_{p}\right\}_{c}$ for any $i, j$. If the sets of all fixed points of $F(z)$ and $G(z)$ coincide with each other except a divisor of order less than one, then $\omega_{1} / \omega_{2}$ is a rational number and $F(z)=G(z)$.

Proof. Define $\tilde{F}(z)=z-F(z)$ and $\tilde{G}(z)=z-G(z)$. If we set $\varphi_{0}(z)=$ $\psi_{0}(z)=z$, they satisfy obviously the conditions (i) $\sim$ (iii). Moreover, (iv) is also satisfied. Because, if $\varphi$ is a non-constant entire function of order less than one such that $\nu_{z} \geqq \nu_{\varphi}$, then we have necessarily $\varphi(z)=c z$ for some $c \in C^{*}$. On the other hand, the assumption implies that $\nu_{\tilde{F}}-\nu_{\tilde{G}}$ is of order less than one. Therefore, by Theorem 7.1, $\omega_{1} / \omega_{2}$ is a rational number and there is a constant $c$ such that

$$
z-\sum_{i=1}^{p} \varphi_{i} f_{j}=c\left(z-\sum_{j=1}^{q} \psi_{i} g_{j}\right)
$$

so that

$$
(c-1) z+\sum_{i=1}^{p} \varphi_{i} f_{i}=c\left(\sum_{j=1}^{q} \psi_{j} g_{j}\right) .
$$

Both sides are regarded as reduced representations of a meromorphic function of $\left(\gamma_{\omega}, \Phi_{0}\right)$-semi-invariant type for some $\omega \in C^{*}$, By Theorem 5.6, we have easily $c=1$. This gives Corollary 7.3.

\section{REFERENCES}

[1] E. Borel, Sur les zéros des fonctions entières, Acta Math., 20 (1897), 357-396.

[2] H. Fujimoto, On families of meromorphic maps into the complex projective space, Nagoya Math. J., 54 (1974), 21-51.

[3] — The uniqueness problem of meromorphic maps into the complex projective space, Nagoya Math. J., 58 (1975), 1-23.

[4] - A uniqueness theorem of algebraically non-degenerate meromorphic maps into $P^{N}(C)$, Nagoya Math. J., 64 (1976), 117-147.

[5] —_ Remarks to the uniqueness problem of meromorphic maps into $P^{N}(\mathrm{C}), \mathrm{I}, \mathrm{II}$, Nagoya Math. J., 71 (1978), 13-41.

[6] - Remarks to the uniqueness problem of meromorphic maps into $P^{N}(C)$, III, Nagoya Math. J., 75 (1979), 71-85.

[ 7 ] M. L. Green, Holomorphic maps into the complex projective space omitting hyperplanes, Trans. AMS., 169 (1972), 89-103. 
[ 8 ] R. Nevanlinna, Le théorème de Picard-Borel et la théorie des fonctions méromorphes, Gauthier-Villars, Paris (1929).

[9] W. Stoll, Ganze Funktionen endlicher Ordnung mit gegebenen Nullstellenflächen, Math. Z., 57 (1953), 211-237.

[10] —- Normal families of non-negative divisors, Math. Z., 84 (1964), 154-218.

[11] H. Urabe and C. Yang, On the zeros of an entire function which is periodic mod a non-constant entire function of order less than one, Proc. Japan Acad. Ser. A. 54 (1978), 142-144.

[12] - On a characteristic property of periodic entire functions, Kodai Math. J., 3 (1979), 253-286.

Nagoya University 